\title{
Mental health attitudes and beliefs in a community sample on the Central Coast in Australia: Barriers to help seeking
}

\begin{abstract}
There continues to be call for greater community awareness actions and strategies to reduce stigma and enhance mental health literacy nationally and internationally. To identify local barriers to help-seeking and perceptions around stigma, we developed a 'mental health attitudes and beliefs' survey which was administered at a range of community events on the Central Coast in New South Wales, Australia. The aim was for the results of this survey to inform the development of strategies that enhance local help-seeking behaviours that are sensitive to the role of age, gender and Indigenous status. People who approached our Mental Health Information stall were invited to complete the survey and 282 individuals completed the survey. The data was analysed descriptively with a focus on comparing subgroups based on age, gender, Indigenous status, and previous service access or experience of mental illness. Cost, stigma and mental health literacy were found to be prominent barriers to help-seeking for the overall cohort; however, the ways in which or extent to which these barriers impact on help seeking varied between subgroups. A discussion of these differences and their implications for practice is the focus of this paper.
\end{abstract}

Keywords: help seeking; youth mental health; service barriers; gender in mental health service access; mental health literacy; mental health stigma 


\section{Introduction}

While it is well established that early detection and treatment of mental health problems can dramatically change the course of their lives, many people continue not to access the mental health care they need (Gulliver, Griffiths, Christensen, \& Brewer, 2012; Wright, McGorry, Harris, Jorm, \& Pennell, 2006). Key barriers to help-seeking include cost, low mental health literacy, and fear of being stigmatised (Reavley, Cvetkovski, Jorm, \& Lubman, 2010; Reavley \& Jorm, 2011; Rickwood, Deane, \& Wilson, 2007; Siu et al., 2012; Wahl, Susin, Lax, Kaplan, \& Zatina, 2012; Wright et al., 2006). There continues to be a call for greater community awareness actions and strategies to reduce stigma and enhance mental health literacy nationally and internationally to help ensure that people receive the care they need (Commonwealth of Australia, 2009b, 2012; Jorm, 2012; Sane Australia, 2013).

In response to this, workers from a youth mental health service on the Central Coast in New South Wales, Australia attend community events to engage the community in mental health literacy and stigma reduction activities. Alongside our belief in the importance of combatting stigma and enhancing mental health literacy, we wondered what other barriers to service access our local community experiences, and how significant mental health stigma and low mental health literacy is locally. To identify barriers to help-seeking and perceptions around stigma, we developed a 'mental health attitudes and beliefs' survey which we administered at a range of community events that we attended. The aim was for the results of this survey to inform the development of strategies to enhance local help-seeking behaviours and that are sensitive to the role of age, gender and Indigenous status. A discussion of the results of this survey is the focus of this paper.

\section{Methods}

The survey was administered at a range of community events that we attended over an 18month period (from December 2014 until May 2015). The service researcher (author 1) developed the survey in consultation with mental health promotion workers to ensure the survey captured information that would be meaningful to them in their role providing community 
awareness activities. The development of the survey was loosely informed by other surveys reported in the literature but was adapted significantly for our own purposes (Reavley \& Jorm, 2011; Siu et al., 2012). The survey questions are outlined in Table 1.

Table 1. Survey questions

The survey was handed out at seven different community events on the Central Coast. Anyone who approached the Mental Health Information stall was invited to complete the survey by one of the mental health workers manning the stall. The surveys were completed either electronically on an iPad or on a hard copy which was then entered manually by the service researcher. We did not record an exact response rate, but the majority of individuals who were invited to complete the survey agreed to do so. Participation was voluntary and anonymous and no names or other identifying information was collected. The sample is not considered representative of the population as the respondents all attended a health promotion community event.

A total of 282 people completed the survey. As per Table 2, more women than men completed the survey, and there was a high representation of people from Aboriginal and/or Torres Straits Islander backgrounds. Ten percent identified as Aboriginal and/or Torres Straits Islander, which is much higher than the 2.3 percent that reside in this area based on census data (Australian Bureau of Statistics, 2012). One of the community events we attended was an Indigenous specific event, National Aboriginal and Islander Observance Committee (NAIDOC) Day, which explains this high rate.

Table 2: Demographics: age groups, gender and Indigenous status of respondents 
The data was analysed descriptively using Microsoft Excel. In addition to presenting the overall results, analysis focussed on comparing subgroups based on age, gender and Indigenous status, and previous service access or experience of mental illness.

\section{Results}

Approximately half $(43.48 \%, \mathrm{n}=120)$ of respondents reported having a current or previous experience with mental illness (Table 3), with an additional ten percent unsure $(10.51 \%, \mathrm{n}=29)$ if they had or had not experienced mental illness. Most respondents knew someone who had experienced a mental health illness/problem $(84.17 \%, \mathrm{n}=234)$, particularly those respondents who had experienced mental illness themselves $(95.83 \%, n=115)$. Only $9.71 \%$ percent $(n=27)$ reported not knowing anyone with a mental illness, and 5.76 percent $(n=16)$ were unsure.

Table 3. Past experiences with mental illness and previous contact with mental health services.

Of those respondents who reported having experienced mental illness, 71.67\% ( $\mathrm{n}=86)$ had previously accessed a mental health services (Table 4). This indicates that 28.33 percent $(n=34)$ of those who reported having a mental illness had never accessed a mental health service, suggesting that they were self-diagnosed.

Table 4. Access to mental health services amongst participants who have experienced mental illness.

Around three quarters of respondents $(74.45 \%, \mathrm{n}=204)$ would access or feel comfortable accessing services for their emotional wellbeing (Table 7). Respondents reported feeling most comfortable talking about their emotional wellbeing to family and friends and least comfortable talking to a GP, followed by psychologist/counsellor (Table 5).

Table 5. "Who would you feel comfortable talking to about your emotional wellbeing?" 
Cost was the most frequently cited barrier to accessing services (41.87\%), closely followed by 'being judged' (38.21\%) (Figure 1). Just over a third of respondents reported that they would be concerned if their friends knew they were seeking support for their emotional wellbeing (34.44\%), and would not tell others that they experience a mental illness (34.93\%) (Table 7). Over half of respondents (51.89\%) agreed that mental health is not talked about by the community (Table 6). Mental health literacy also appears to be a barrier; nearly twelve percent $(11.48 \%)$ agreed or strongly agreed with the statement that "people who are weak or overly sensitive let mental illness affect them" (Table 7).

Figure 1. "What concerns you most about getting help from a health professional?"

Table 6. Participants who agreed that "Mental illness is not talked about by..."

Table 7. What do you think about mental health? Beliefs around accessing services, mental health literacy and mental health stigma.

Analysis indicates that experiences and beliefs around accessing services, mental health literacy and mental health stigma may vary based on age, gender, Indigenous status, and previous service access.

\section{The role of age}

Young people aged 18-25 reported the highest rates of mental health problems $(67.50 \%, \mathrm{n}=27)$ (Table 3). Out of those respondents who reported having or having experienced mental health issues, young people aged 12-17 were least likely to have accessed a mental health service (48.65\%, $\mathrm{n}=18)$ (Table 4). Age also plays a role in the type of emotional supports respondents felt comfortable accessing. Respondents' level of comfort accessing professional support increased with age, at least up to the age of fifty (Table 5). Young people aged 12-17 and 18-24 
were most comfortable getting support from their family or friends, and least comfortable accessing professional help. Less than half of young people aged 12-17 agreed that they would access mental health services (Table 7).

In terms of barriers to accessing services, the greatest concerns reported by 12-17 year olds were 'embarrassment' and 'being judged' (Figure 1). This cohort was more concerned about these two factors than any other age group, followed by Indigenous respondents. Respondents aged 12-17 were also most likely to be worried if their friends knew they were getting support for their emotional wellbeing (Table 7). Young people aged 12-17 also reported the highest rates of concerns around confidentiality. Respondents aged 12-17 also reported lower mental health literacy (Table 7).

Middle aged respondents, in particular those aged 36-50, reported the least barriers to help seeking and were the most likely to agree that they would access mental health services at 95.92 percent (Table 7). This percentage increases with age, but drops again for respondents over fifty to 88.89 percent.

Respondents over fifty reported discomfort getting support for their emotional wellbeing, and were least comfortable talking to their family and friends about their emotional wellbeing. They report high levels of perceived stigma, and are most likely to agree that mental illness is not talked about by the community (78.79\%), their family (63.64\%) and their friends (51.61\%) (Table 6), and that mental illness is not accepted by society (75.68\%) (Table 7).

Despite this perception of mental illness as stigmatised, they do not report embarrassment as a key barriers to help seeking (Figure 1).

\section{The role of gender}

Female respondents were more likely than male respondents to report experience with mental illness (48.35\% vs $34.04 \%$ ) (Table 3). Of those respondents who reported having experienced mental illness, more females than males $(80.68 \%$ vs $46.88 \%)$ had accessed a mental health service (Table 4). While less likely to access services, male respondents reported feeling more comfortable talking about their emotional wellbeing to family, a GP and a psychologist than 
female respondents (Table 5). Only their comfort levels talking to friends were on par with female respondents.

Female respondents were more concerned about embarrassment and being judged than male respondents (Figure 1). They were also much more likely to agree that mental illness is not accepted by society than male respondents $(51.11 \%$ vs $26.88 \%)$ (Table 7$)$. However, despite viewing mental illness as less stigmatised than females, males were more likely to report that they would not tell others that they suffered from a mental health problem (40.66\% vs $32.04 \%)$. Gender did not appear to influence the likelihood of knowing someone with a mental health illness/problem (females $85.79 \%$ vs males $81.05 \%$ ).

\section{The role of indigenous status}

Nearly half $(47.62 \%, \mathrm{n}=10)$ of Aboriginal and/or Torres Strait Islander respondents reported having experienced mental health problems/illness, which is higher than the overall cohort (Table 3). Despite the high levels of mental illness in this group, Aboriginal and/or Torres Strait Islander respondents reported significantly lower levels of help-seeking than the overall cohort. Compared to the general cohort, they were far less likely to agree that they would access mental health services $(55.56 \%$ vs $70.57 \%)$ and this group was the least likely to agree that they would encourage family and friends to seek help (66.67\% vs 92.62\%) (Table 7). While Aboriginal and/or Torres Strait Islander respondents reported being as comfortable as the overall cohort in talking to their family, they were less comfortable talking to friends or professional services (Table 5).

In terms of barriers to help seeking, mental health stigma and poor mental health literacy seems to be of particular concern for this cohort. Aboriginal and/or Torres Strait Islander respondents are nearly as likely as respondents over the age of fifty to agree that mental illness is not talked about by their family $(60.00 \%)$ or their friends $(52.63 \%)$ (Table 6), and not accepted by society (61.90\%) (Table 7). They were less likely to report knowing someone with a history of mental illness than the overall cohort (63.64\% vs $84.17 \%$ ) (Table 3), which can 
perhaps be attributed to mental illness not being talked about as much within the Indigenous community.

Aboriginal and/or Torres Strait Islander respondents were three times as likely to agree with the statement "Only people who are weak and overly sensitive let mental illness affect them ", and almost half of Indigenous respondents agreed that their peers often made jokes about mental illness compared to less than a third of the overall cohort (Table 7). They rated 'embarrassment' and 'being judged' as a barrier nearly as much as young people aged 12-17 (Figure 1).

\section{The role of previous service access}

Those respondents who had previously accessed mental health services were far less likely to be comfortable talking to their families and friends about their emotional wellbeing compared to the overall cohort and to people who had never accessed mental health services (Table 5), and more comfortable speaking to GPs and psychologists/ counsellors. This may also indicate that accessing services increases someone's comfort doing so. Figure 1 shows that those respondents who had previously accessed services are less likely to identify embarrassment as a barrier to service access than those who have not. People with a history of mental illness themselves were also more likely to know somebody who had experienced mental illness than those without a history of mental illness (95.83\% vs $75.61 \%)$.

\section{Discussion}

Consistent with the literature the overall findings indicate that for the current respondents cost, stigma and low mental health literacy were key barriers to help-seeking (Coates \& Howe, 2014; Corrigan, 2004; Siu et al., 2012; Vogel, Bitman, Hammer, \& Wade, 2013; Wahl et al., 2012). The way in which or extent to which these barriers may impact on help seeking appear to vary based on age, gender, Indigenous status, and previous service access. 


\section{The role of age}

\section{Young people}

Our findings support previous observation that young people are most likely to experience mental health problems and least likely to access professional help (Kessler et al., 2012; Marcus, Westra, Eastwood, \& Barnes, 2012; McGorry, Purcell, Goldstone, \& Amminger, 2011; Patulny, Muir, Powell, Flaxman, \& Oprea, 2013; Plaistow et al., 2014; Reavley et al., 2010; Yap, Reavley, \& Jorm, 2012). Consistent with the literature, young people reported fear of being judged and embarrassment (Coates \& Howe, 2014; Marcus et al., 2012) confidentiality (Plaistow et al., 2014), and low mental health literacy (Ross, Hart, Jorm, Kelly, \& Kitchener, 2012; Yap \& Jorm, 2012) as key barriers to getting support. While it makes sense that the youngest cohort of respondents in this sample have had the least service access (as young people have not had as much opportunity to access services as older people), it remains concerning that half of young people who report mental health problems have not sought professional help.

We echo previous calls for youth friendly services to be more visible and awareness campaigns to encourage young people to access support earlier (Gibb, Fergusson, \& Horwood, 2010; Gulliver, Griffiths, \& Christensen, 2010; Howe, Batchelor, Coates, \& Cashman, 2013; Plaistow et al., 2014; Wright et al., 2006; Yap et al., 2012). While professional supports play an important role in the provision of mental health care, consistent with other studies, our findings highlight the important role of families and friends in this space (Coates, 2016; Jorm, 2012). Young respondents are more comfortable talking to their friends and family than professional services (Coates \& Howe, 2014; Leavey, Rothi, \& Rini, 2011; Ross et al., 2012), and we propose that family and friends should be supported and equipped with the mental health literacy to support each other. It is increasingly recognised that young people as well as parents need to be better equipped with the necessary skills and knowledge to support each other through mental health problems (Jorm, 2012; Jorm \& Kitchener, 2011; Ross et al., 2012; Yap \& Jorm, 2012), and more resources and actions in this space are needed so young people can more 
effectively support each other and receive the help they need without having to access specialist services.

Improved mental health literacy will better equip young people to support each other, can help address concerns around confidentiality and may also reduce levels of embarrassment and discomfort accessing mental health support (Coates \& Howe, 2014; Marcus et al., 2012). Furthermore, a range of strategies could be put in place to help reduce young people's level of embarrassment and discomfort accessing services, such as providing them with detailed information around what to expect from mental health services prior to appointments (Plaistow et al., 2014).

\section{Older people}

Respondents over fifty reported most discomfort getting support, and were least comfortable talking to their family and friends about their emotional wellbeing is. Consistent with previous observations, they reported a stigmatised view of mental illness, as only affecting people who are 'weak' and 'overly sensitive'. Stigma and negative attitudes towards mental health care are reasons older Australians fail to access mental health care, preferring to handle mental illness on their own or not accepting that they may be affected (Kantor, Knefel, \& Lueger-Schuster, 2017; Muir-Cochrane, O'Kane, Barkway, Oster, \& Fuller, 2014). Perceptions of mental health stigma may explain the lower rates of mental illness reported by older respondents.

In terms of barriers to help seeking, despite high rates of perceived stigma, older respondents were less likely to identify embarrassment as a key barrier than younger people. Young people reported fear of being judged and embarrassment as key barriers even though they viewed mental illness as less stigmatised than older people. While this observation is not surprising as it is well recognised that young people are more sensitive to the views of society and their peer groups, it is of note that older people identified cost and confidentiality as more important help seeking barriers. The barriers and enablers of help seeking for older people is not well understood, at least not in comparison to young people, and this is an important area of future research. Despite high rates of mental illness, including suicide, in older people (Conwell, 
Van Orden, \& Caine, 2011; World Health Organisation, 2014), much of the help seeking literature is focused on young people, with little research into the barriers and enablers to help seeking for older people (Hom, Stanley, \& Joiner Jr, 2015).

To enhance help seeking in this population, strategies that combat stigmatised perceptions of mental illness and encourage informal supports may be most appropriate for this population. The importance of informal supports, such as encouragement of close family and friends (Kantor et al., 2017), is increasingly highlighted as critical enabler to help-seeking, and our findings indicate that older people struggle to utilise friends and family as sources of emotional support.

\section{The role of gender}

Female respondents were more likely than male respondents to report experience with mental illness. While this is not consistent with lifetime prevalence rates which indicate that men are more likely to experience mental illness than women (Australian Bureau of Statistics, 2008), it is consistent with the tendency of women to seek help and disclose experience of mental illness more than men (Australian Bureau of Statistics, 2008; Australian Institute of Health and Welfare, 2016; Harding \& Fox, 2015; Patulny et al., 2013). Even though, as expected, females were more likely to access services and disclose experience of mental illness than males, they reported feeling more uncomfortable seeking help and viewed society as more stigmatised than males. While there is evidence that indicates that males are more vulnerable to mental health stigma than females (Clement et al., 2015; Oliffe et al., 2016), our findings suggest a complicated relationship between gender, perceptions of stigma and help seeking.

While males view society as less stigmatised and reported higher levels of comfort talking about emotional wellbeing, they were less likely to seek support or disclose mental health problems to others. Females viewed society as more stigmatised, felt more uncomfortable accessing services and were more concerned about embarrassment and being judged, but despite this they were more likely to seek formal or informal supports than males. It is possible that men 'feel more comfortable' accessing services but don't see the need to do so or don't believe 
that it would be helpful. Females may be more likely to access services even if they feel uncomfortable doing so.

This finding suggests that the type of health promotion strategies that may be effective in increasing help-seeking behaviour for females may be different from males. While for females strategies that increase comfort may be effective, these strategies may be less effective for males. Males may experience other barriers to service access not identified by the current study, such as a lack of confidence that treatment will be effective. Strategies that raise awareness of the effectiveness and purpose of interventions may be of greater benefit to males compared to strategies increasing their level of comfort seeking help. A lack of understanding of what mental health care entails has been previously reported by men as a barrier to help-seeking (Harding \& Fox, 2015). Interventions or awareness campaign that seek to shift men's perception of social norms around mental illness may also be effective in enhancing help seeking (Harding \& Fox, 2015). Males are highly influenced by the attitudes and behaviours of their peers in terms of help seeking and strategies that normalise help-seeking have been found effective in increasing service access for men (Harding \& Fox, 2015).

\section{The role of Indigenous Status}

A higher percentage of Aboriginal and/or Torres Strait Islander respondents reported personal experience with mental health problems than the overall cohort, consistent with the high rates of mental illness experienced by this populations (of between fifty and 75 percent) (Jorm, Bourchier, Cvetkovski, \& Stewart, 2012). Aboriginal and/or Torres Strait Islander respondents reported high levels of mental health stigma, and they were least likely to seek help or encourage others to do so.

These observations are not new, and it is well recognised that Aboriginal and/or Torres Strait Islander people are less likely to access mainstream health services than the general population in proportion to their need for a range of reasons including a history of racism and discrimination and resultant lack of trust in mainstream services, negative past experiences with 'mainstream' health, low mental health literacy, mental health stigma, shame in seeking help 
and lack of culturally appropriate services (Commonwealth of Australia, 2007, 2009a;

Department of Health and Ageing, 2013; Isaacs, Pyett, Oakley-Browne, Gruis, \& Waples-

Crowe, 2010; Sherwood \& Edwards, 2006; South Australia Deptartment of Health, 2010;

Westerman, 2010; Williamson et al., 2010).

Despite what seems to be an obvious need for mental health awareness campaigns to specifically target this population, this remains a neglected area of study. There is a dearth of research assessing whether programmes designed to improve Indigenous mental health literacy result in increased service access (Isaacs et al., 2010). The model of care that applies to the nonIndigenous population differs to that required by the Indigenous population, who have a holistic view of health and different ways of managing illness within their communities (Isaacs et al., 2010). The Indigenous community is also an extremely heterogeneous group, and therefore locally relevant collaborative research is required to inform locally-delivered care (Isaacs et al., 2010). In order to increase utilisation of mental health services by the Indigenous population, participatory and inclusive practices are crucial (Farnbach et al., 2017; Povey et al., 2016). Online programmes may also be an effective way to provide information and support to the Indigenous community, and online and telephone services have been successfully delivered to Indigenous people in the past (Povey et al., 2016). Furthermore, support groups, including Alcoholics Anonymous, have also been well received in Indigenous communities (McIntyre et al., 2017), and it be may valuable to identify which component of support groups work well for Indigenous communities, so these can be translated into mental health care for this population more broadly.

\section{The role of previous service access}

Our findings indicate that those respondents who had previously accessed mental health services were far less likely to be comfortable talking to their families and friends about their emotional wellbeing, and more comfortable speaking to GPs and psychologists/counsellors. This may indicate that people who access services are uncomfortable getting support from their friends and families or have fewer personal relationships they can draw on for support. In 
addition, it may indicate that accessing services increases someone's comfort doing so. It is well established that having a positive past experience accessing services is a facilitator of help seeking (Gulliver et al., 2010; Rickwood, Deane, Wilson, \& Ciarrochi, 2005; Smith, 2012). Furthermore, our findings indicate that people with a mental illness were more likely to know somebody with a mental illness, and this may mean that mental health is more likely to be normalised among their peers, enabling help-seeking behaviours (Harding \& Fox, 2015).

\section{Limitations and area for future research}

The study's sample size is small and cannot be generalised. While the study made some interesting observations around the differences between the different cohorts, these should be interpreted with caution given the small sample size of each subgroup, and also given that the sample sizes of these subgroups are not equal. However, while the sample is not considered representative of the wider population, approximately half of respondents reported having a current or previous experience with mental illness, which is consistent with estimates that nearly half (45.5\%) of Australians will experience a mental health problem at some point in their life (Australian Bureau of Statistics, 2008).

The respondents all attended a community event and approached a Mental Health Information stall and the findings should be interpreted with this in mind. Our sample may have greater mental health awareness and needs than the general population. For example, the high level of service access reported by the current sample can most likely be attributed to the sampling strategy. Approximately seventy percent of individuals who reported having experienced mental illness had accessed a mental health services, which is significantly higher than estimates that between a third and half of people with mental illness access professional help (Australian Bureau of Statistics, 2014-2015; Gulliver et al., 2012).

Given the small sample size, further research is required to test these findings quantitatively, and unpack them further qualitatively. Specifically, we call for further research into the role of gender in help seeking. The female respondents in this study were almost twice as likely as male respondents to agree that mental illness is not accepted by society and were 
less comfortable seeking professional help for their mental health, and yet were more likely to do so. While we can theorise on why this is and suggest that perhaps men have less faith in the effectiveness of treatment, further research is required. This study highlighted that the relationship between comfort accessing services and actually seeking help is not straightforward, and this should be explored further.

\section{Conclusion}

Addressing stigma and low mental health literacy is a key priority identified by many government plans and actions and can have a dramatic impact on the lives of people with mental illness. In order to develop effective strategies to address these concerns, an understanding of barriers to help-seeking behaviours is crucial. This study helped us better understand the barriers preventing community members from accessing mental health services by uncovering local perceptions and attitudes around help seeking.

Different key concerns and perceptions were observed by analysing responses based on age groups, gender and Indigenous status. Identifying these differing barriers to service access can help inform the development of specific approaches better suited to different subgroups. For young people, this study highlights the important role of families and friends, suggesting that bolstering informal support networks may lessen the need for and demand on formal support services for this population. Older respondents as well as Aboriginal and/or Torres Strait islander respondents reported the strongest perceptions of mental health stigma, suggesting that mental health literacy strategies that seek to combat this negative perception may be most appropriate for these cohorts. Gender also appears to play an important role in help-seeking, and this study highlights a need for further research in this space.

\section{Compliance with Ethical Standards}

Ethics approval for this study was received through the Hunter New England Human Research Ethics Committee and the Central Coast Local Health District Research Office. All procedures performed in studies involving human participants were in accordance with the ethical standards 
of the institutional and/or national research committee and with the 1964 Helsinki declaration and its later amendments or comparable ethical standards. 


\section{References}

Australian Bureau of Statistics. (2008). National survey of mental health and well-being. Summary of results Cat No 4364.0. Retrieved from Canberra:

Australian Bureau of Statistics. (2012). 2011 Census of Population and Housing, Basic Community Profile (Catalogue number 2001.0): Central Coast. Retrieved from http://www.abs.gov.au/websitedbs/censushome.nsf/home/communityprofiles?opendocu $\underline{\text { ment } \& \text { navpos }=230}$

Australian Bureau of Statistics. (2014-2015). National Health Survey Australia - First Results. Australian Institute of Health and Welfare. (2016). Mental health services - in brief 2016. Cat. no. HSE 180. Canberra.

Clement, S., Schauman, O., Graham, T., Maggioni, F., Evans-Lacko, S., Bezborodovs, N., . . . Thornicroft, G. (2015). What is the impact of mental health-related stigma on helpseeking? A systematic review of quantitative and qualitative studies. Psychological Medicine, 45, 11-27.

Coates, D. (2016). Client and parent feedback on a Youth Mental Health Service: The importance of family inclusive practice and working with client preferences. International Journal of Mental Health Nursing.

Coates, D., \& Howe, D. (2014). The importance and benefits of youth participation in mental health settings from the perspective of the headspace Gosford Youth Alliance in Australia Children and Youth Services Review, 46, 294-299. doi:DOI: 10.1016/j.childyouth.2014.09.012

Commonwealth of Australia. (2007). NSW Aboriginal Mental Health and Well Being Policy $2006-2010$

Australian Government Retrieved from http://www0.health.nsw.gov.au/policies/pd/2007/pdf/PD2007 059.pdf.

Commonwealth of Australia. (2009a). Cultural Respect \& Communication Guide: A NSW Aboriginal Sexual Health Resource. 
Commonwealth of Australia. (2009b). Fourth National Mental Health Plan: An agenda for collaborative government action in mental health 2009-2014. Retrieved from

Commonwealth of Australia. (2012). Work Wanted: Mental health and workforce participation. Retrieved from

Conwell, Y., Van Orden, K., \& Caine, E. D. (2011). Suicide in Older Adults. Psychiatric Clinics of North America, 34(2), 451-468.

Corrigan, P. (2004). How stigma interferes with mental health care. American Psychologist, 59(7), 614-625.

Department of Health and Ageing. (2013). National Aboriginal And Torres Strait Islander Health Plan 2013-2023 Australian Government,.

Farnbach, S., Eades, A., Fernando, J. K., Gwynn, J. D., Glozier, N., \& Hackett, M. L. (2017). The quality of Australian Indigenous primary health care research focusing on social and emotional wellbeing: A systematic review. Public Health Research and Practice, 27(4), e27341700.

Gibb, S. J., Fergusson, D. M., \& Horwood, L. J. (2010). Burden of psychiatric disorder in young adulthood and life outcomes at age 30. The British Journal of Psychiatry, 197, 122-127.

Gulliver, A., Griffiths, K. M., \& Christensen, H. (2010). Perceived barriers and facilitators to mental health help-seeking in young people: a systematic review. BMC Psychiatry, $2010(10), 113$

Gulliver, A., Griffiths, K. M., Christensen, H., \& Brewer, J. L. (2012). A systematic review of help-seeking interventions for depression, anxiety and general psychological distress. BMC Psychiatry, 12, 81 .

Harding, C., \& Fox, C. (2015). It's not about "Freudian couches and personality changing drugs": An investigation into men's mental health help-seeking enablers. American Journal of Men's Health, 9(6), 451-463.

Hom, M. A., Stanley, I. H., \& Joiner Jr, T. E. (2015). Evaluating factors and interventions that influence help-seeking and mental health service utilization among suicidal individuals: A review of the literature. Clinical Psychology Review, 40, 28-39. 
Howe, D., Batchelor, S., Coates, D., \& Cashman, E. (2013). Nine key principles to guide Youth Mental Health: Development of Service Models in New South Wales. Early Intervention in Psychiatry, 8(2), 190-197. doi:10.1111/eip.12096

Isaacs, A. N., Pyett, P., Oakley-Browne, m., Gruis, H., \& Waples-Crowe, P. (2010). Barriers and facilitators to the utilisation of adult menta; health services by Australia's Indigenous people: Seeking a way forwward. International Journal of Mental Health Nursing, 19, 75-82.

Jorm, A. (2012). Mental Health Literacy: Empowering the Community to Take Action for Better Mental Health. American Psychologist, 67(3), 231-243.

Jorm, A., Bourchier, S. J., Cvetkovski, S., \& Stewart, G. (2012). Mental Health of Indigenous Australians: a review of findings from community surveys. Med J Aust, 196, 118-121. Jorm, A., \& Kitchener, B. A. (2011). Noting a landmark achievement: Mental Health First Aid Training Reaches 1\% of Australian Adults. Australian and New Zealand Journal of Psychiatry, 45, 808-813.

Kantor, V., Knefel, M., \& Lueger-Schuster, B. (2017). Perceived barriers and facilitators of mental health service utilization in adult trauma survivors: A systematic review. Clinical Psychology Review, 52, 52-68.

Kessler, R. C., Avenevoli, S., Costello, J. E., Georgiades, K., Green, J. G., Gruber, M. J., . . Merikangas, K. R. (2012). Prevalence, Persistence, and Sociodemographic Correlates of DSM-IV Disorders in the National Comorbidity Survey Replication Adolescent Supplement. Arch Gen Psychiatry, 69(4), 372-380.

Leavey, G., Rothi, D., \& Rini, P. (2011). Trust, autonomy and relationships: the help-seeking preferences of young people in secondary level schools in London (UK). Journal of Adolescence, 34, 685-693.

Marcus, M. A., Westra, H. A., Eastwood, J. D., \& Barnes, K. L. (2012). What are young people saying about mental health? An analysis of internet blogs. Journal of Medical Internet Research, 14(1), e17. 
McGorry, P., Purcell, R., Goldstone, S., \& Amminger, G. (2011). Age of onset and timing of treatment for mental and substance use disorders: implications for preventive intervention strategies and models of care. Current Opinion in Psychiatry, 24(4), 301306.

McIntyre, C., Harris, M. G., Baxter, A. J., Leske, S., Diminic, S., Gone, J. P., . . Whiteford, H. (2017). Assessing service use for mental health by Indigenous populations in Australia, Canada, New Zealand and the United States: A rapid review of population surveys. Health Research Policy and Systems, 15, 67.

Muir-Cochrane, E., O'Kane, D., Barkway, P., Oster, C., \& Fuller, J. (2014). Service provision for older poeple with mental health problems in a rural area of Australia. Aging and Mental Health, 18(6), 759-766.

Oliffe, J. L., Orgodniczuk, J. S., Gordon, S. J., Creighton, G., Kelly, M. T., Black, N., \& Mackenzie, C. (2016). Stigma in male depression and suicide: a Canadian sex comparison study. Community Mental Health Journal, 52, 302-310.

Patulny, R., Muir, K., Powell, A., Flaxman, S., \& Oprea, I. (2013). Are we reaching them yet? Service access patterns among attendees at the headspace youth mental health initiative Child and Adolescent Mental Health, 18(2), 95-102.

Plaistow, J., Masson, K., Koch, D., Wilson, J., Stark, R., Jones, P., \& Lennox, B. (2014). Young Poeple's views of UK mental health services Early Interv Psychiatry, 8, 12-23.

Povey, J., Mills, P. P. J. R., Dingwall, K. M., Lowell, A., Singer, J., Rotumah, D., . . Nagel, T. (2016). Acceptability of mental health apps for Aboriginal and Torre Strait Islander Australians: A qualitative study. Journal of Medical Internet Research, 18(3), e65.

Reavley, N., Cvetkovski, S., Jorm, A., \& Lubman, D. I. (2010). Help seeking for substance use, anxiety and affective disorders among young people: results from the 2007 Australian National Survey of Mental Health and Wellbeing. 44, 729-735.

Reavley, N., \& Jorm, A. (2011). Stigmatizing attitudes towards people with mental disorders: findings from an Australian National Survey of Mental Health Literacy and Stigma. Australian and New Zealand Journal of Psychiatry, 45, 1086-1093. 
Rickwood, D., Deane, F. P., \& Wilson, C. (2007). When and how do young people seek professional help for mental health problems? Medical Journal of Australia, 187, S35S39.

Rickwood, D., Deane, F. P., Wilson, C., \& Ciarrochi, J. (2005). Young people's help-seeking for mental health problems. Australian e-Journal for the Advancement of Mental Health (AeJAMH), 4(3), 1-34.

Ross, A. M., Hart, L. M., Jorm, A. F., Kelly, C. M., \& Kitchener, B. A. (2012). Development of key messages for adolescents on providing basic mental health first aid to peers: A Delphi consensus study. Early Interv Psychiatry, 6, 229-238.

Sane Australia. (2013). A life without stigma. Retrieved from

Sherwood, E., \& Edwards, T. (2006). Decolonisation: A critical step for improving Aboriginal health. Contemp Nurse, 22(2), 178-190.

Siu, B., Chow, K., Lam, L., Chan, W., Tang, V., \& Chiu, W. (2012). A questionnaire survey on attitudes and understanding towards mental disorders. East Asian Arch Psychiatry, 22, $18-24$.

Smith, C. (2012). Mental health and help-seeking in adolescence. Australasian Epidemologist, $19(1), 5-8$.

South Australia Deptartment of Health. (2010). Aboriginal Health Care Plan 2010-2016. South Australia: Dept. of Health, Statewide Service Strategy Division.

Vogel, D. L., Bitman, R. L., Hammer, J. H., \& Wade, N. G. (2013). Is stigma internalized? The longitudinal impact of public stigma on self-stigma. Journal of Counseling Psychology, 60(2), 311-316.

Wahl, O., Susin, J., Lax, A., Kaplan, L., \& Zatina, D. (2012). Knowledge and Attitudes About Mental Illness: A Survey of Middle School Students. Psychiatric Services, 63(7).

Westerman, T. G. (2010). Engaging Australian Aboriginal Youth in Mental Health Services. . Australian Psychologist, 45(3), 212-222. 
Williamson, A. B., Raphael, R., Redman, S., Daniels, J., Eades, S. J., \& Mayers, N. (2010). Emerging themes in Aboriginal child and adolescent mental health: findings from a qualitative study in Sydney, New South Wales. Medical Journal of Australia, 192(10).

World Health Organisation. (2014). Preventing suicide: A global imperative. Geneva.

Wright, A., McGorry, P., Harris, M., Jorm, A., \& Pennell, K. (2006). Development and evaluation of a youth mental health community awareness campaign - the Compass strategy. BMC Public Health, 6(215).

Yap, M. B., \& Jorm, A. (2012). Young people's mental health first aid intentions and beliefs propectively predict their actions: Findings from an Australian National Survey of Youth. Psychiatry Research, 196, 315-319.

Yap, M. B., Reavley, N., \& Jorm, A. (2012). Australian youth still have limited awareness of headspace: Results from a national survey. Australian \& New Zealand Journal of Psychiatry, 46(1), 28-34. 